NASA/TM-1999-209184

AIAA-99-0748

\title{
A Comparison of Three Navier-Stokes Solvers for Exhaust Nozzle Flowfields
}

Nicholas J. Georgiadis, Dennis A. Yoder, and James R. DeBonis

Glenn Research Center, Cleveland, Ohio

Prepared for the

37th Aerospace Sciences Meeting \& Exhibit

sponsored by the American Institute of Aeronautics and Astronautics

Reno, Nevada, January 11-14, 1999

National Aeronautics and

Space Administration

Glenn Research Center

May 1999 


\section{Acknowledgments}

The authors would like to thank Khaled Abdol-Hamid and Steven Massey of AS\&M, John Carlson and Paul Pao of NASA Langley Research Center, and Kevin Early of General Electric Aircraft Engines for assistance in learning to use the PAB Navier-Stokes code.

Available from

NASA Center for Aerospace Information

7121 Standard Drive

Hanover, MD 21076

Price Code: A03
National Technical Information Service 5285 Port Royal Road Springfield, VA 22100

Price Code: A03 


\title{
A COMPARISON OF THREE NAVIER-STOKES SOLVERS FOR EXHAUST NOZZLE FLOWFIELDS
}

\author{
Nicholas J. Georgiadis ${ }^{*}$, Dennis A. Yoder ${ }^{\dagger}$ and James R. DeBonis ${ }^{\dagger}$ \\ National Aeronautics and Space Administration \\ Glenn Research Center \\ Cleveland, Ohio 44135
}

\begin{abstract}
A comparison of the NPARC, PAB, and WIND (previously known as NASTD) Navier-Stokes solvers is made for two flow cases with turbulent mixing as the dominant flow characteristic, a twodimensional ejector nozzle and a Mach 1.5 elliptic jet. The objective of the work is to determine if comparable predictions of nozzle flows can be obtained from different Navier-Stokes codes employed in a multiple site research program. A single computational grid was constructed for each of the two flows and used for all of the Navier-Stokes solvers. In addition, similar $k-\varepsilon$ based turbulence models were employed in each code, and boundary conditions were specified as similarly as possible across the codes. Comparisons of mass flow rates, velocity profiles, and turbulence model quantities are made between the computations and experimental data. The computational cost of obtaining converged solutions with each of the codes is also documented. Results indicate that all of the codes provided similar predictions for the two nozzle flows. Agreement of the Navier-Stokes calculations with experimental data was good for the ejector nozzle. However, for the Mach 1.5 elliptic jet, the calculations were unable to accurately capture the development of the three dimensional elliptic mixing layer.
\end{abstract}

\section{Introduction}

In the High Speed Research (HSR) program, the National Aeronautics and Space Administration (NASA) and its aerospace industry partners have been developing the necessary technologies for a future commercial supersonic passenger transport. One major focus of the HSR program is the collaborative development of an exhaust nozzle which reduces jet noise at take-off while maintaining high thrust levels. The currently favored nozzle

*Research Engineer. Nozzle Branch. Senior Member AlAA.

$\doteqdot$ Research Engineer. Nozzle Branch. Member AIAA. design is a two-dimensional mixer-ejector, which mixes the high speed flow from the engine core (primary flow) with entrained external flow (secondary flow). The mixed flow exits the nozzle at a lower jet velocity and hence produces less noise than an equivalent unsuppressed nozzle. In addition. high thrust is maintained due to the ejector action.

Reynolds-averaged Navier-Stokes solvers have been used extensively by members of the NASA-industry team to analyze candidate mixerejector nozzle configurations. However, with a different Reynolds-averaged Navier-Stokes (referred to hereafter as "Navier-Stokes") solver used by each of the participating organizations, it is not clear that results obtained at multiple sites are comparable. As an example, consider that one site obtains flow predictions (thrust, extent of mixing, etc.) for a given nozzle that are different from predictions obtained at another site for a second nozzle. The question will arise as to whether the differences are due to code issues (different Navier-Stokes codes or code settings), realistic flow physics, or a combination. As a result, it is necessary to understand the capabilities and differences of these codes in order to use results from different Navier Stokes codes in a single research program such as this HSR nozzle development effort.

The results of a previous study (Ref. 1) indicated that similar Navier-Stokes predictions could be obtained from different organizations for two baseline flows, a supersonic round jet and compressible planar shear layer. However, other HSR studies in which more geometrically complex nozzle configurations were examined (also involving multiple organizations) indicated a broader range in results. One such study, described in Ref. 2, was a transonic boattail drag study in which the predicted boattail drag varied by over a factor of two from one organization to another for some of the cases examined, even with using the same computational 
grid and boundary conditions. Another is a more recent study where several members of the HSR NASA-industry team obtained predictions for a large scale mixer-ejector nozzle, in which there were significant differences in predicted thrust levels and pumping ratios

In the current study, three general purpose Navier-Stokes solvers that have been employed to analyze complex nozzle configurations for the HSR nozzle program (and which are accessible to the authors) are compared for two nozzle cases that have flow features representative of mixer ejector nozzles (i.e. turbulent mixing is the dominant flow characteristic). They are a two-dimensional ejector nozzle and a supersonic elliptic nozzle exiting into ambient air. Comparisons of code predictions to experimental data are made for each of the nozzle flow cases to determine relative accuracy of the three codes. In addition, comparisons of code speed and other factors affecting usage are made herein. For each of the nozzle cases considered, a common computational grid was used for each of the flow solvers, although the grid blocking structure for each code was different to enable the most efficient usage of each code. In addition, the $k-\varepsilon$ (or equivalent) turbulence models in each code that are generally used for most mixer nozzle applications with that code are employed for the calculations obtained here. No compressibility or other corrections to the turbulence models were used in this study. A brief description of the three codes examined in this study is presented in the next section, followed by discussion of the nozzle flow cases and results.

\section{Navier-Stokes Solvers}

NPARC: The NPARC code is a Navier-Stokes solver that has been jointly supported by NASA Glenn Research Center and U.S. Air Force Arnold Engineering Development Center through the NPARC Alliance (Ref. 3). NPARC solves the Navier-Stokes equations discretized in a central finite difference form, which requires the use of explicit artificial viscosity for stability. Several turbulence models are available ranging from algebraic to two-equation models. The Chien low Reynolds number $k-\varepsilon$ turbulence model (Ref. 4) was used for the mixing studies investigated here. These $k$ and $\varepsilon$ equations are solved uncoupled from the Navier-Stokes equations using an upwind biased solver.
PAB: PAB is a Navier-Stokes code originally developed and supported by NASA Langley Research Center (Refs. 5-7). Recently, the responsibility for development and support of PAB was transferred to AS\&M, Inc., with the primary code author still serving as the main developer. PAB solves the Navier-Stokes and turbulence equations discretized in finite volume form. The default third order upwind Roe scheme was employed for the calculations obtained here, although other schemes such as that due to Van Leer are available. Grid sequencing is available in PAB, which allows for fast initial flowfield development using a computational grid with a fraction of the points of the final grid (i.e. every other point in each computational direction). Several two-equation models are available in PAB, including algebraic Reynolds stress formulations. In the current study, the low Reynolds number $k-\varepsilon$ turbulence model due to Launder and Sharma (Ref. 8) was used.

WIND (NASTD): The WIND Navier-Stokes solver is a general purpose Navier-Stokes solver that is the new production flow solver of the NPARC Alliance (Ref. 9). The code has its foundation in the NASTD code (Refs. 10 and 11 ) developed and used by the McDonnell Douglas Corporation (now BoeingSt.Louis), and has recently been modified to include some features of the NPARC and NXAIR (Ref. 12) codes. Several options for the discretization of the Navier-Stokes equations are available, with a modified second order Roe scheme for stretched grids (described in Ref. 11) used for these nozzle studies. Of the several turbulence models available in WIND, two low Reynolds number models were employed for this study. The first is the Menter two equation model (commonly referred to as the SST model) which is a hybrid $k-\omega / k-\varepsilon$ two-equation model with $k-\omega$ used in near wall regions and $k-\varepsilon$ used away from the wall and in free shear layer regions (Refs. 13,14). The second is the Chien $\mathrm{k}-\varepsilon$ model, which was incerporated into WIND similarly to the model available in NPARC.

\section{$\underline{\text { Results }}$}

\section{Two-Dimensional Ejector Nozzle}

The first nozzle case examined in this study is a two-dimensional ejector nozzle tested by Gilbert and Hill (Ref. 15). A schematic of the ejector nozzle test case is shown in Fig. 1. This flow is dominated by mixing of the primary flow with 
entrained secondary flow and is similar in fundamental operation to nozzles that have been investigated in the HSR program. In the experiment, a suction system was used in the sidewalls to keep the flows as two-dimensional as possible by eliminating the blockage due to the sidewall boundary layers.

A grid having 131 points in the axial (flow) direction by 121 points in the vertical direction was constructed for use with each of the three codes. The results of Refs. 16 and 17 showed that a computational grid having approximately 100 axial grid points in similar mixing layers was sufficient to provide solutions that did not change with additional grid points. In particular, Ref. 17 indicated that grids having 131 and 251 points in the axial direction produced identical results for this ejector nozzle flow using NPARC with the Chien $k-\varepsilon$ turbulence model. The grid used here was packed to solid surfaces such that the first point off of the wall corresponded to a $y+$ between $I$ and 2, depending on the local flow conditions. For the two-dimensional version of NPARC, the single block grid with no modifications to the two-dimensional grid structure was used. For PAB and WIND, the grid was first broken into three blocks for the primary inflow, secondary inflow, and mixing regions. In addition, since single versions of PAB and WIND are used for both two and three dimensional cases, a third grid dimension was added having two points for PAB and one point for WIND. Because the geometry of the nozzle is symmetric about a plane passing through the center of the ejector nozzle assembly, only one half of the ejector nozzle was modeled for all codes.

Wherever possible, default settings for each of the three codes were used. A section later in this report will discuss particular findings of this study in which non-standard code settings were required to obtain solutions. Boundary conditions were also specified in a manner as similar as possible with all three codes. Atmospheric pressure was specified at the ejector inflow while the total pressure specified for the primary nozzle was set to provide the nozzle pressure ratio set in the experiment (nozzle total pressure divided by atmospheric static pressure) to 2.44. The outflow pressure was the static pressure measured in the experiment at approximately 10.5 inches downstream of the primary nozzle exit. The total temperature of the primary nozzle was set to $644 \mathrm{R}$ while the ambient temperature $(550 \mathrm{R})$ was specified for the secondary inflow. These total temperatures were specified exactly with NPARC and PAB using a subsonic inflow (also known as free) boundary condition. The WIND code employs a characteristic-based boundary condition for inflows which does not enable exact specification of the total temperature. However, the maximum variation of the total temperature from that desired at the primary and secondary inflows was less than one degree Rankine.

Velocity profiles obtained from the calculations are compared to experimental data in Fig. 2. The axial positions were measured relative to the primary nozzle exit plane and the vertical positions are measured relative to the centerline. The latter were nondimensionalized by the local half-duct height $H$. The velocity profiles at 3, 5, 7, and 10.5 inches downstream of the primary nozzle exit plane indicate that the predictions from the four cases are quite similar throughout the mixing section, although the PAB (k- $\varepsilon$ ) and WIND (SST) results indicate slightly lower peak velocities than the NPARC $(k-\varepsilon)$ and WIND $(k-\varepsilon)$ solutions that were obtained with the Chien model. Profiles of turbulent viscosity, shown in Fig. 3, also indicate higher turbulence levels predicted by the PAB $(k-\varepsilon)$ and WIND (SST) solutions for this case. Mass flow rates were integrated across the primary and secondary flow paths using each of the four converged solutions and are compared to experimental data from Ref. 15 in Table 1 . The resulting pumping ratios (primary mass flow rate divided by secondary mass flow rate) from these solutions are very similar to each other but slightly lower than that indicated by the experimental data.

Table 1. Mass Flow Information for TwoDimensional Ejector Nozzle Calculations

\begin{tabular}{lrrr}
\hline \multicolumn{1}{c}{ Case } & $\begin{array}{c}\text { Primary } \\
\text { Rass Flow } \\
\text { Rate lbm/s) }\end{array}$ & \multicolumn{2}{c}{$\begin{array}{c}\text { Secondary } \\
\text { Mass Flow }\end{array}$} \\
Rate lbm/s) & $\begin{array}{c}\text { Pumping } \\
\text { Ratio }\end{array}$ \\
\hline NPARC k- $\varepsilon$ & 0.718 & 3.09 & 4.30 \\
PAB k- $\varepsilon$ & 0.711 & 3.10 & 4.36 \\
WIND k-E & 0.713 & 3.13 & 4.39 \\
WIND SST & 0.713 & 3.14 & 4.40 \\
Experiment & 0.707 & 3.30 & 4.67
\end{tabular}

The number of iterations to obtain a converged solution and the total CPU hours used were determined as follows for each code. The first requirement was that the $\mathrm{L} 2$ residual error was required to drop by at least 4 orders of magnitude for each case. Secondly, the velocity at all points along the nozzle centerline was monitored from one set of 
iterations to the next, with a set typically consisting of 500-1000 iterations. Each case was run until all centerline velocities did not change by more than 0.001 percent between sets, and we could assume that a final answer had been reached. The "converged solution" point was then found by examining centerline velocities from previous sets, and finding the first solution in which all centerline velocities were within 0.01 percent of the final values.

Table 2 provides convergence information for the four solutions obtained for this twodimensional ejector nozzle case. It should be noted that for this case, the NPARC solution had the advantage of having been run with the NPARC2D code, which does not have the additional computational overhead associated with the three dimensional codes, while the PAB and WIND solutions were obtained with the single version of each of these codes that is used for both two and three dimensional cases. Since most realistic nozzle configurations have three dimensional characteristics, it will be more meaningful to examine the convergence information for the next case, an elliptic nozzle, which required three dimensional calculations with all codes. In addition, full advantage of the grid sequencing capability of $\mathrm{PAB}$ was not made, since we monitored flow quantities on the finest grid to determine convergence. Only the first 1000 iterations of the PAB solution were made with a grid having one half the number of points of the baseline grid in each computational direction.

Table 2. Convergence Information for TwoDimensional Ejector Nozzle Calculations

\begin{tabular}{lrr}
\multicolumn{1}{c}{ Case } & Iterations & \multicolumn{1}{c}{ CPU hours } \\
(SGI R 10000)
\end{tabular}

\section{Elliptic Nozzle}

The elliptic nozzle examined in this study was that tested by Seiner and Ponton (Ref. 18). A schematic of the convergent-divergent nozzle is shown in Fig. 4 and had an exit area of $1.571 \mathrm{in}^{2}$ with an aspect ratio (major axis diameter divided by minor axis diameter) of 2 . The nozzle was operated at $564 \mathrm{R}$ total temperature and a total pressure corresponding to 3.67 times the freestream static pressure (to provide the design Mach number of 1.5) and exited into ambient air. These total conditions were used to specifiy inflow boundary conditions for each code as was done for the ejector nozzle case. The surrounding ambient air was modeled as having a freestream Mach number of 0.01 to prevent convergence difficulties.

Due to geometric symmetry along the major and minor axes, only a 90 degree section of the nozzle was modeled in this study. A three dimensional grid having 121 points in the axial direction, 91 points in the radial direction (going outward from the jet centerline), and 25 points in the circumferential direction (spanning through the 90 degree segment from the major axis symmetry plane to minor axis symmetry plane) was constructed. This grid was packed to solid surfaces such that the first point off of the wall corresponded to a $y+$ between 1 and 2 , as was done for the two-dimensional ejector nozzle. A single block grid (with appropriate internal boundaries) was used with the NPARC code. For PAB, the grid was split into three blocks for the internal nozzle, freestream inflow, and plume regions. For WIND, the grid structure was further modified to split the plume block into four blocks (for a total of six grid blocks). This was done to enable the WIND code to run efficiently with multiple processors.

In the figures referred to in the following discussion, all positions are normalized by the nozzle exit area equivalent diameter and velocities are normalized by the ideal jet exit velocity. A comparison of the jet centerline velocity decay is shown in Fig. 5. The initial decay of the NPARC solution is somewhat faster than the other solutions and experimental data, but further downstream, the rate of decay of all four solutions approaches the same asymptotic level. Figure 6 compares velocity profiles from the calculations to experimental data at several axial locations along the plane of the major axis. The velocity profiles from the calculations are very similar, except for the lower peak velocity of the NPARC solution at $X / D_{e 4}=7.6$ and 10.1. Figure 6 shows that none of the velocity profiles obtained from the calculations matches the experimental data well in terms of capturing the details of the shear layer growth. As a result, examining only the centerline velocity decay results in Fig. 5 may lead to the incorrect conclusion that the Navier-Stokes calculations are accurately modeling the flow found in the experiment. 
Turbulent viscosity profiles along the plane of the major axis at two axial positions in the shear layer, $\mathrm{X} / \mathrm{D}_{\mathrm{eq}}=3.8$ and $\mathrm{X} / \mathrm{D}_{\mathrm{cq}}=10.1$, are shown in Fig. 7. It is interesting to note that the two solutions obtained with the same turbulence model in different codes (the Chien $k-\varepsilon$ model in NPARC and WIND) predicted very similar development of turbulent viscosity. This was also the case for the twodimensional ejector nozzle flow, as shown in Fig. 3. Velocity and turbulent viscosity profiles along the plane of the minor axis are shown in Figs. 8 and 9 respectively. The behavior of the solutions is very similar to that observed along the major axis, with the NPARC solution having a lower peak velocity at $\mathrm{X} / \mathrm{D}_{\mathrm{cy}}=7.6$ and 10.1 than the other solutions. Further examination of the velocity profiles at $\mathrm{X} / \mathrm{D}_{\mathrm{cu}}=10.1$ in Figs. 6(d) and 8(d) indicates that the calculations predict a shear layer thinner than the experimental data along the major axis, but wider than the experimental data along the minor axis. That is, the calculations all predict that the initially elliptic jet becomes more round downstream of the nozzle exit than that indicated by experimental data. The inability of the codes to correctly capture the three dimensional effects of this elliptic jet may likely be due to the isotropic turbulence models used for all of the calculations.

The same convergence criteria described in the previous section for the two-dimensional ejector nozzle case was used here for the elliptic nozzle. This information is provided in Table 3. Substantial differences between the CPU requirements for these three dimensional calculations and those of the previous two-dimensional case may be observed. In particular, NPARC took substantially longer than the other codes to obtain a converged solution for the three dimensional elliptic nozzle. As mentioned in the previous section, NPARC required the least $\mathrm{CPU}$ resources for the two-dimensional ejector nozzle case because only NPARC has a separate code for two-dimensional calculations. For both of the nozzle cases discussed in this report. NPARC required the most iterations to oblain a converged solution. It should also be noted that the reported NPARC CPU requirements are the total $C P U$ hours required while using six processors, and the WIND CPU hours are from using three processors, both on an SGI Power Challenge computer. A multiple processor version of the $P A B$ code was released during the time of this study, but was not used for these nozzle calculations. As was done for the ejector nozzle case, a grid having one half the number of grid points of the final grid in each computational direction was used for the first 1000 iterations of the PAB calculation.

Table 3. Convergence Information for Elliptic Nozzle Calculations

\begin{tabular}{lrr}
\hline \multicolumn{1}{c}{ Case } & Iterations & $\begin{array}{c}\text { CPU hours } \\
\text { (SGI R 10000) }\end{array}$ \\
\hline NPARC k- $\varepsilon$ & 35000 & 404 \\
PAB k- $\varepsilon$ & 9000 & 110 \\
WIND k- $\varepsilon$ & 22000 & 161 \\
WIND SST & 17000 & 114
\end{tabular}

\section{Code-Specific Findings}

In this section, some additional code issues that arose during this study are presented.

NPARC: Unlike the PAB and WIND codes, the NPARC code uses central differencing for the inviscid fluxes, which requires explicit artificial viscosity for code stability. NPARC uses a variation of the Jameson technique for computing artificial viscosity (Ref. 19) Although minimizing the levels of artificial viscosity is desirable for code accuracy, some artificial viscosity is usually necessary for obtaining a converged solution. As a result, an incompressible flat plate flow having extensive experimental data available from Wieghardt (Ref. 20) was examined to determine the effects of settings for the second and fourth coefficients of artificial viscosity (termed DIS2 and DIS4, respectively) on turbulent flow predictions. A comparison of velocity profiles did not indicate any noticeable differences among five solutions obtained by varying the coefficients from DIS2 $=0.25$ and DIS4 $=0.64$ (the NPARC default values) to DIS2 = 0.00 and DIS4 $=0.00$ (no artificial viscosity). However, a comparison of skin friction along the flat plate in Fig. 10 shows that variation of the second and fourth artificial viscosity coefficients has an effect on the near wall behavior.

For the nozzle flows investigated in the study, the lowest levels of artificial viscosity which enabled converged solutions to be obtained were DIS2 $=0.10$ and DIS4 $=0.30$. An NPARC solution obtained for the elliptic nozzle flow with DIS2 $=0.25$ and DIS4 $=0.64$ produced a velocity profile at the nozzle exit with a slightly lower core flow velocity than that obtained with DIS2 $=0.10$ and DIS4 $=0.30$. In the region where the elliptic jet mixed with ambient air, however, no noticeable differences were observed. 
PAB: In becoming familiar with the PAB code, we first examined the same flat plate flow used to evaluate NPARC, in order to determine the effects of input settings unique to the PAB code. Figure 11 presents the results of varying the freestream lower limits of turbulent viscosity (normalized by the reference molecular viscosity) and turbulence intensity for this flat plate flow while using the Roe inviscid flux splitting scheme. It may be observed in Fig. 11 that using the default values for these quantities (turbulent viscosity ratio $=0.10$ and turbulent intensity $=.001$ ) results in a boundary layer profile that differs substantially from solutions obtained with higher turbulent viscosity ratio limits and the experimental data. Further examination of the quantities near the boundary layer edge indicated an abrupt change in the mean flow and turbulent quantities to the freestream values, instead of the more gradual change from the upper portion of the boundary layer into the freestream. In response, the main author of PAB suggested that the results obtained with the low turbulent viscosity limit were due to the low levels of implicit artificial viscosity associated with the Roe flux splitting scheme.

Varying these turbulence model limiters in a similar manner for the nozzle cases discussed previously in this report did not result in as large of an effect on the boundary layer profiles in the isolated flows upstream of the mixing regions, nor in the mixing regions. However, further examination of the effects of the PAB turbulent viscosity ratio and turbulence intensity limits may be warranted. particularly for determining effects of these settings on the complicated wall boundary layers within mixer ejector inlets.

WIND (NASTD): One issue that arose with the WIND code was the treatment of turbulent flow quantities at block interfaces. Figure 12 shows turhulent viscosity along the centerline obtained with the WIND code using both the Chien $k-\varepsilon$ and SST turbulence models, with the symbols representing the turbulent viscosity values at the exact grid point locations. One of the block boundaries for the WIND calculations was located at $X / D_{i y}=24.6$. That is, the computational plane at this streamwise location is the interface of two blocks in the mixing section. It may he observed that the turbulent viscosity at $\mathrm{X} / \mathrm{D}_{\mathrm{cu}}=24.6$ and the first point upstream are identical. although the surrounding points for both the Chien $k-\varepsilon$ and SST solutions have a gradient through this region. This behavior is due to the zero-order extrapolation of the turbulence variables at an outflow block boundary. It may be more accurate to use a higher order extrapolation, especially for block interfaces where large gradients in the turbulence model quantities are expected.

A second issue with the WIND code was the inability to specify the total temperature exactly at an inflow. The boundary conditions used most frequently at inflows using NPARC and PAB operate the same in that total pressure and total temperature are specified as inputs and then are held constant throughout the calculations. With WIND, the boundary condition used for such inflows is known as an arbitrary inflow, in which characteristic variables (including total pressure but not total temperature) are held constant at an inflow. For the nozzle calculations discussed in this report, the inflow quantities specified with WIND were specified carefully to try to match the total temperature used with NPARC and PAB, but it was not possible to have an exact match at all of the inflow boundary points.

\section{Summary of Results}

In this study, three Navier-Stokes solvers (NPARC, PAB, and WIND-formerly NASTD) used by members of different organizations were investigated at one site for two high speed nozzle flows - a two-dimensional ejector nozzle and an elliptic nozzle. The objective was to determine the relative capabilities and differences of these codes when using similar two-equation turbulence models and a common baseline computational grid. The major observations from this study may be grouped into two categories, the first being accuracy of the code solutions when compared to experimental data and the second of relative performance of the codes, including computational cost.

All of the codes were able to predict the two-dimensional ejector nozzle flow relatively accurately. The calculations seemed to overpredict the peak velocity at axial locations close to the primary nozzle exit, but the agreement of all of the solutions with experimental data improved downstream. In addition, all of the calculations indicated somewhat lower ejector pumping ratios than that obtained from the experimental data.

Comparisons of calculations with experimental data for the elliptic nozzle case indicated that none of the codes was able to accurately model the three dimensional mixing layer. In particular, all of the calculations indicated that 
the initially elliptic jet (with a $2: 1$ major axis to minor axis ratio) became round much faster than indicated by experimental data. This observation was made only after examining the velocity profiles along the major and minor axes; examining only the centerline velocity behavior may lead to the incorrect conclusion that the agreement of all of the solutions with experimental data was good. This failure of the codes to predict the three dimensional behavior of the elliptic jet is most likely due to the limitations of the two-equation turbulence models used with each code, all of which assume a locally isotropic turbulent state.

In comparing calculations from the different codes to each other for both the ejector nozzle and elliptic nozzle cases, all of the solutions provided similar predictions of mean flow shear layer mixing quantities, and for the ejector nozzle, similar predictions of mass flow rates in the primary and secondary streams. From the profiles of turbulent viscosity for both nozzle flows, the most interesting observation was that the Chien $k-\varepsilon$ model installed in NPARC and WIND operated very similarly. To compare computational costs using each of the codes, it is probably only meaningful to consider the three dimensional calculation of the elliptic jet; as the two-dimensional ejector case run with NPARC used the version of the code specifically constructed for two-dimensional cases. For the three dimensional elliptic nozzle case, PAB and WIND provided converged solutions at a significantly lower cost than NPARC (measured in CPU hours on an SGI Power Challenge), and in significantly fewer iterations. Finally, a section following the ejector and elliptic nozzle results provided some findings specific to each of the three codes including necessary modifications to standard code inputs and issues for further investigation.

\section{Concluding Remarks}

In considering all of these observations, we conclude that it should be possible to obtain relatively similar results across organizations using different favored Navier-Stokes codes, as was the general conclusion of Ref.1. However, the results discussed in this study were obtained after taking several steps to operate the codes as similarly as possible, including using the same computational grid for each code, using similar turbulence models with all special corrections (i.e. for compressibility) turned off, and post-processing the converged solutions with a single technique. It was also necessary to take into account code features unique to each of the flow solvers, and make necessary adjustments when possible to obtain comparable results.

The code-specific features found during the course of this study can affect solutions to varying extents and likely do not represent a complete list of differences between codes. They should, however, emphasize the importance of continually identifying such code differences and constructing a formalized set of inputs and other settings for each of the codes used, in order to obtain results from different codes that will be comparable in a single research program. Continual validation of the codes used in single research program against a set of specific benchmark test cases which have three dimensional flow features representative of more realistic configurations is also recommended, especially with the frequent release of new versions of productionuse Navier-Stokes solvers like NPARC, PAB, and WIND.

Two issues regarding post-processing should also be addressed. The first is that for finite volume codes that store flow information at the cell centers (like PAB) instead of at the actual node points, care must be taken in transferring flow quantities from cell centers back to the computational grid points, or the reverse as the case may be. The second is that post-processing techniques used at different sites may also be responsible for differences in reported results, and efforts should be undertaken to identify such discrepancies.

The results of this and other studies have shown that two-equation turbulence models such as $k-\varepsilon$ have definite limitations when applied to realistic three dimensional flows. No special corrections or sets of constants for these models have shown to be universally better for such complex flow problems. Unfortunately, more sophisticated turbulence models such as full Reynolds stress models are neither likely to be readily installed in production-use Navier-Stokes solvers, nor are they guaranteed to provide substantially more accurate solutions (Refs. 21 and 22). Advanced techniques such as large eddy simulations may hold promise for improved predictions several years in the future. Until these advances are made, however, we will probably be limited to two-equation turbulence models and need to continue to determine their limitations and range of applicability. 


\section{References}

1. Barber, T.J., Chiappetta, L.M., DeBonis, J.R., Georgiadis, N.J., and Yoder, D.A., "An Assessment of Parameters Influencing Prediction of Shear Layer Mixing," AIAA Paper 97-2639, July 1997.

2. DeBonis, J.R., Georgiadis, N.J., and Smith, C.F., "Validation of the NPARC Code for Nozzle Afterbody Flows at Transonic Speeds," AIAA Paper 95-2614, July 1995.

3. Power, G.D., Cooper, G.K., and Sirbaugh, J.R., "NPARC 2.2 - Features and Capabilities," AIAA Paper 95-2609, July 1995.

4. Chien, K.-Y., "Predictions of Channel and Boundary Layer Flows with a Low-Reynolds-Number Turbulence Model," AIAA Journal. Vol. 20, no. 1, January 1982, pp. 33-38.

5. Abdol-Hamid, K.S., "A Multiblock/Multizone Code (PAB3D-v2) for the Three-Dimensional NavierStokes Equations: Preliminary Applications," NASA CR-182032, October 1990.

6. Abdol-Hamid, K.S., Uenishi, K., and Turner, W., "Three-Dimensional Upwinding Navier-Stokes Code for k-_ Model for Supersonic Flows," AIAA Paper 91-1669, June 1991.

7. Abdol-Hamid, K.S., Carlson, J.R., \& Pao, S.P., "Calculation of Turbulent Flows Using Mesh Sequencing and Conservative Patch Algorithm," AIAA Paper 95-2336, July 1995.

8. Launder, B.E., and Sharma, B.I., "Application of the Energy-Dissipation Model of Turbulence to the Calculation of Flow Near a Spinning Disc," Letters in Heat and Mass Transfer. Vol. 1, 1974, pp. 131-138.

9. Bush. R., Power, G., and Towne, C.. "WIND: The Production Flow Solver of the NPARC Alliance," AIAA Paper 98-0935, January 1998.

10. Bush, R.H., "A Three Dimensional Zonal NavierStokes Code for Subsonic Through Hypersonic Propulsion Flowfields." AIAA Paper 88-2830, July 1988 .

11. Cain, A.B. and Bush, R.H., "Numerical Wave Propagation Analysis for Stretched Grids." AlAA Paper 94-()172. January 1994.
12. Tramel, R.W., and Nichols, R.H., "A Highly Efficient Numerical Method for Overset-Mesh Moving-Body Problems," AIAA Paper 97-2040, June 1997.

13. Menter, F.R., "Zonal Two Equation k- $\omega$ Turbulence Models for Aerodynamic Flows," AIAA Paper 93-2906, July 1993.

14. Mani, M., Ladd, J.A., Cain, A.B., and Bush, R.H., "An Assessment of One- and Two-Equation Turbulence Models for Internal and External Flows," AIAA Paper 97-2010, June 1997.

15. Gilbert, G.B., and Hill, P.G., "Analysis and Testing of Two-Dimensional Slot Nozzle Ejectors With Variable Area Mixing Sections, NASA CR-2251, May 1973.

16. Georgiadis, N.J., Dudek, J.C., and Tierney, T.P., "Grid Resolution and Turbulent Inflow Boundary Condition Recommendations for NPARC Calculations," AIAA Paper 95-2613, July 1995.

17. Georgiadis, N.J. and Yoder, D.A., "Use of NavierStokes Methods for the Calculation of High-Speed Nozzle Flow Fields," AIAA Paper 94-3212, June 1994.

18. Seiner, J.M., and Ponton, M.K., "Supersonic Acoustic Source Mechanisms for Free Jets of Various Geometries," AGARD 78th Specialists Meeting on Combat Aircraft Noise, Bonn, Germany, October 1991.

19. Jameson, A., Schmidt, W., and Turkel, E., "Numerical Solutions of the Euler Equations by Finite Volume Method for Solving the Euler and Navier-Stokes Equations for High Speed Flows," AIAA Paper 81-1259, June 1981.

20. Wieghardt, K., and Tillman, W., "On the Turbulent Friction Layer for Rising Pressure," NASA TM 1314, 1951.

21. Bushnell, D., "Turbulence Modeling in Aerodynamic Shear Flow - Status and Problems," AIAA Paper 91-0214, January 1991.

22. Birch, S.F., "The Role of Structure in Turbulent Mixing." AIAA Paper 97-2636, July 1997. 


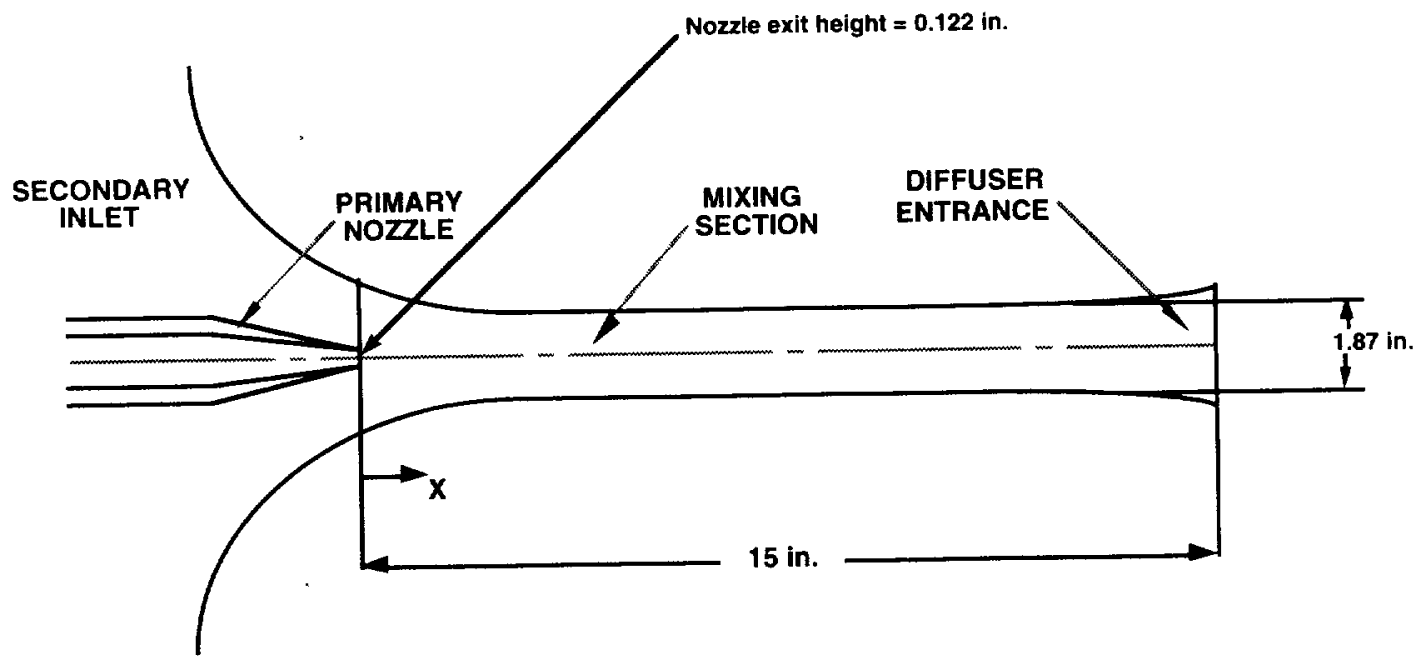

Fig. 1. Schematic of two-dimensional ejector nozzle test case.

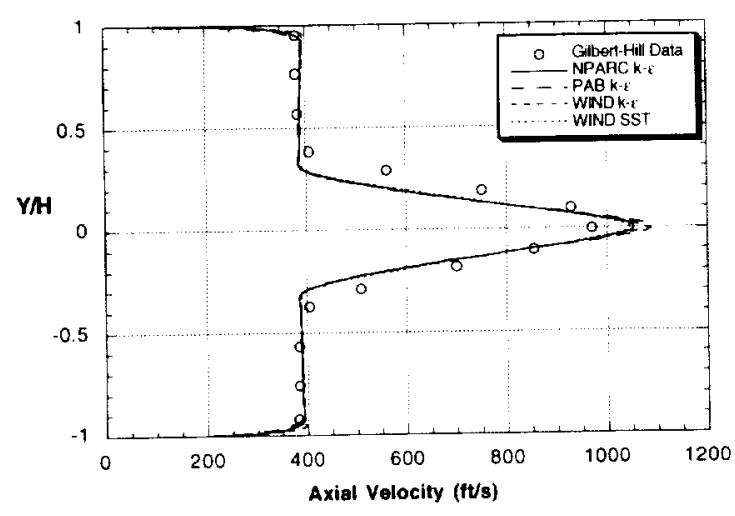

(a) $X=3$ in.

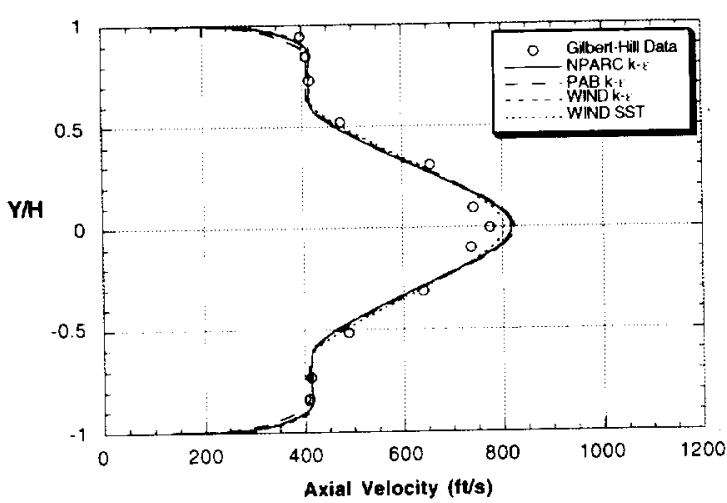

(c) $X=7$ in.

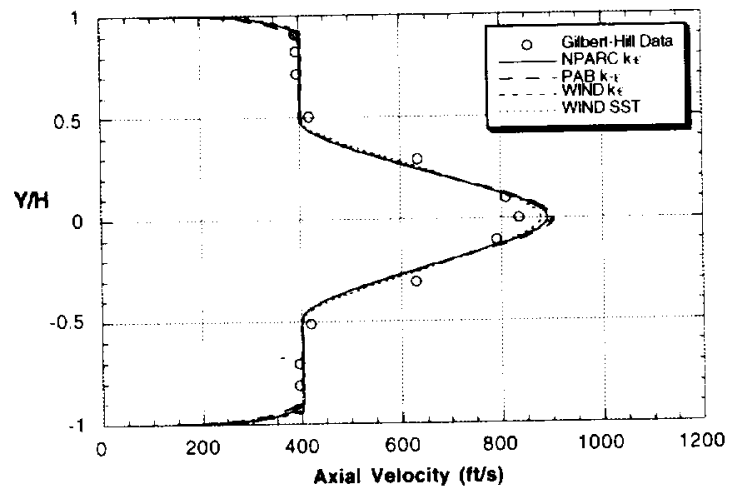

(b) $X=5$ in.

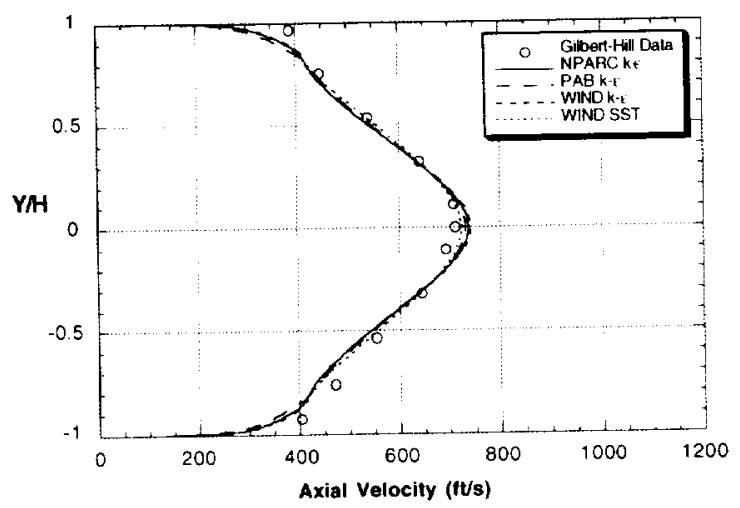

(d) $X=10.5$ in.

Fig. 2. Velocity profiles through mixing section for 2D ejector nozzle. 


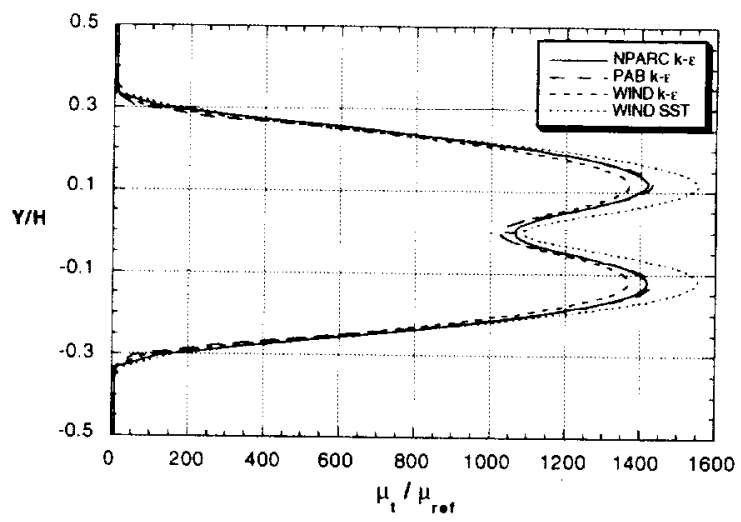

(a) $X=3$ in.

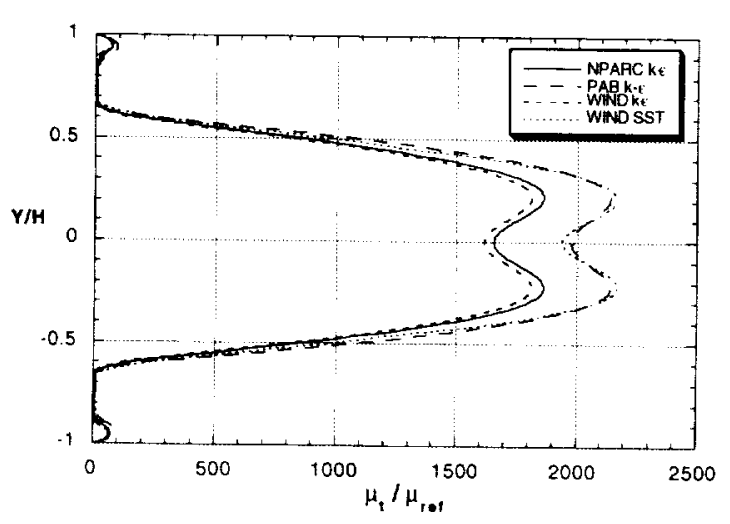

(c) $X=7$ in.

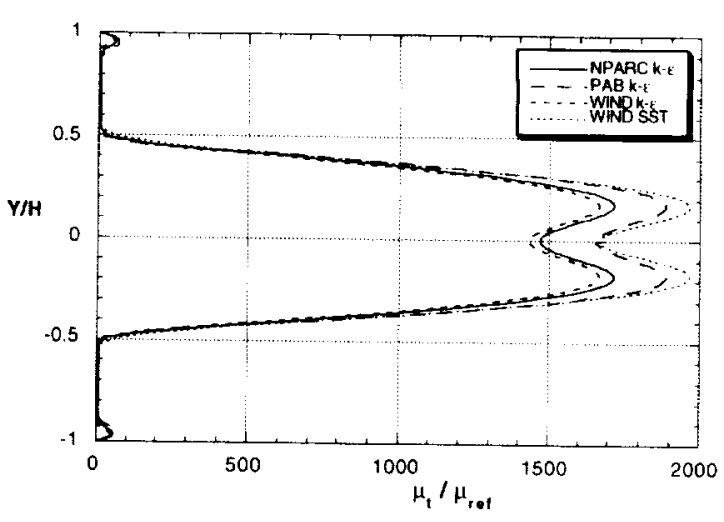

(b) $X=5$ in.

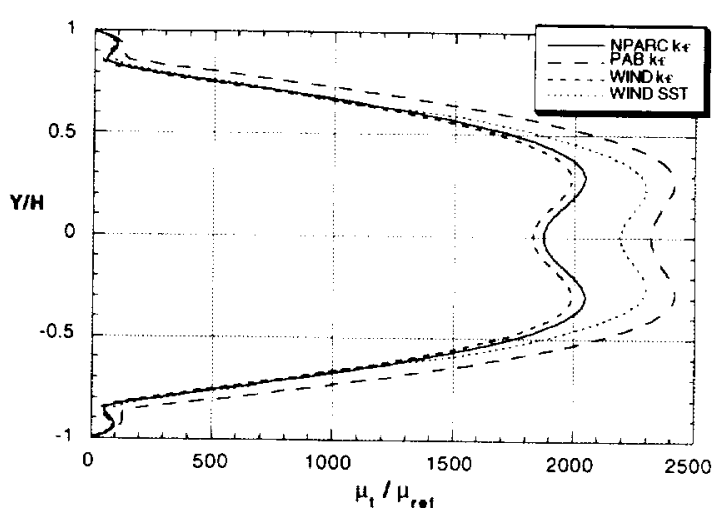

(d) $X=10.5$ in.

Fig. 3. Turbulent viscosity profiles through mixing section for $2 \mathrm{D}$ ejector nozzle.

Ambient Air (Modeled as Mach 0.01 Flow)

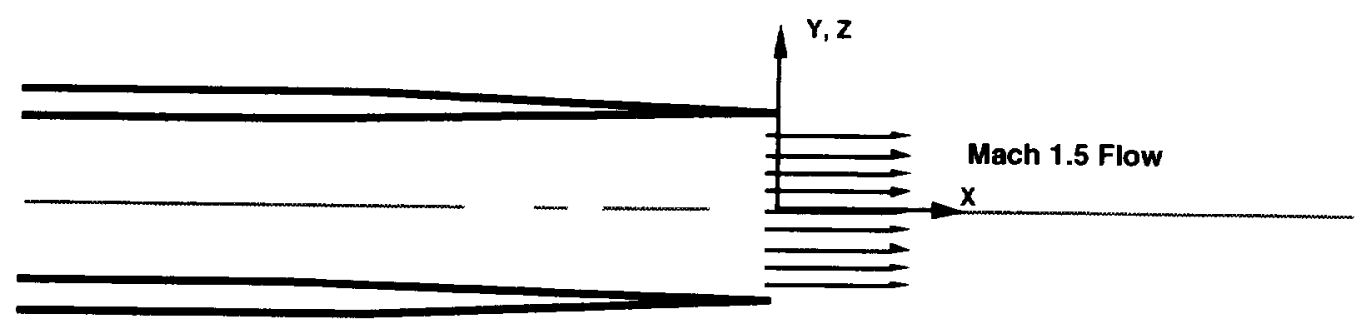

Fig. 4. Schematic of elliptic nozzle test case. 


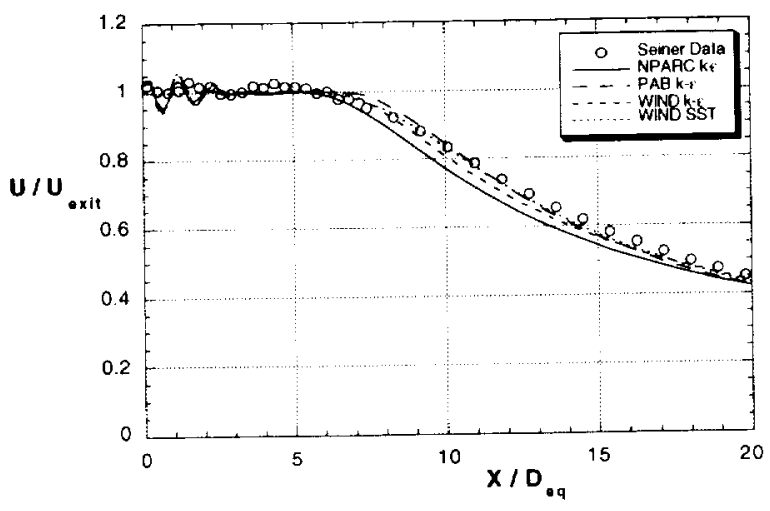

Fig. 5. Centerline velocity decay for elliptic nozzle.

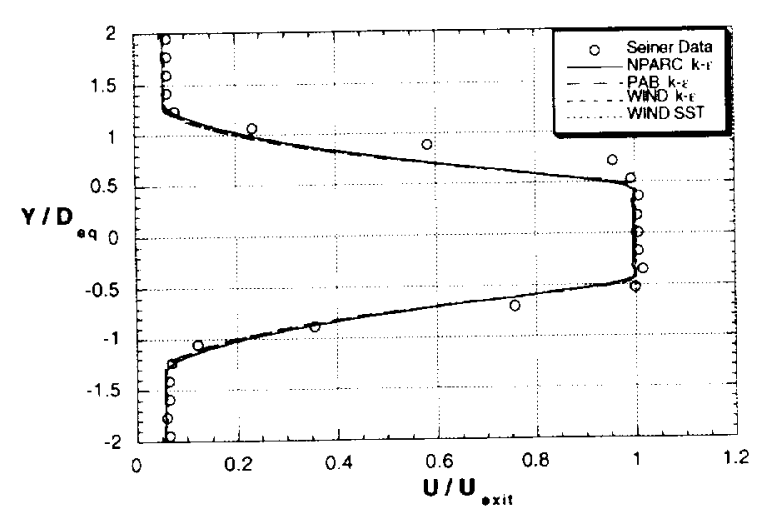

(a) $\mathrm{X} / \mathrm{D}_{\mathrm{i} 4}=3.8$.

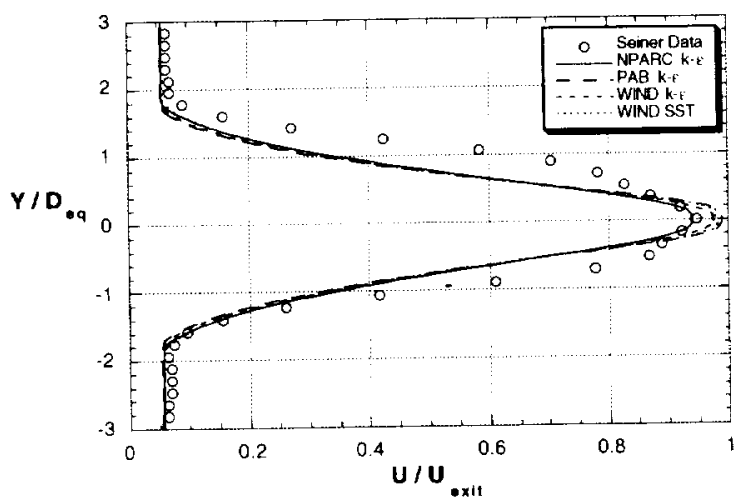

(c) $\mathrm{X} / \mathrm{D}_{\mathrm{cu}}=7.6$.

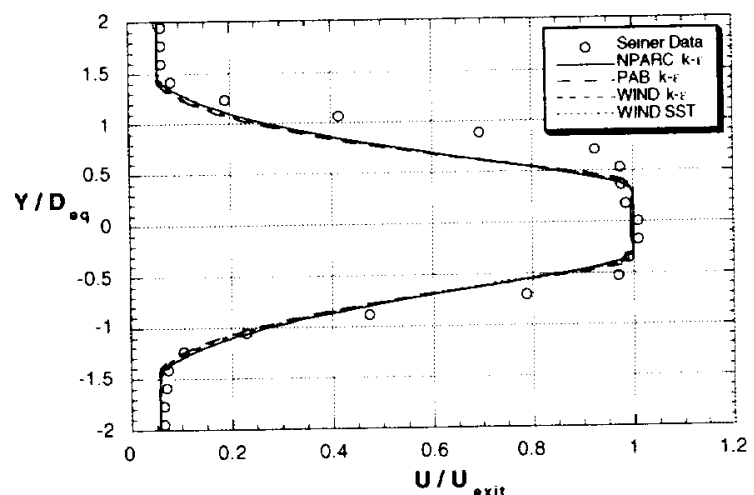

(b) $\mathrm{X} / \mathrm{D}_{\mathrm{cu}}=5.1$.

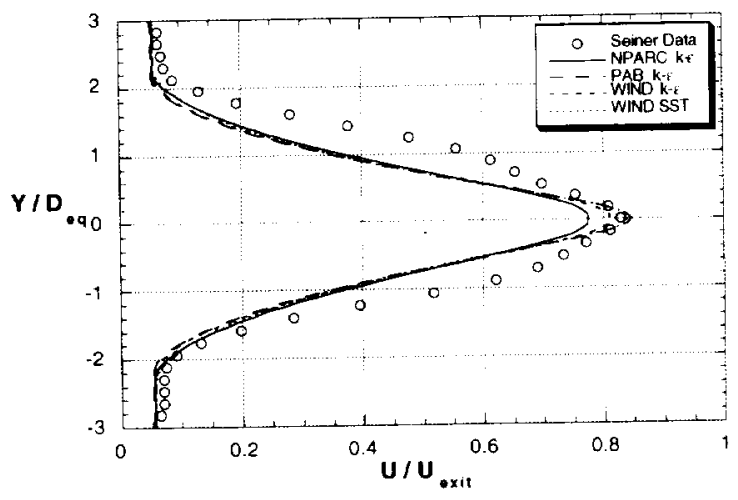

(d) $X / D_{e y}=10.1$.

Fig. 6. Velocity profiles along major axis for elliptic nozzle. 


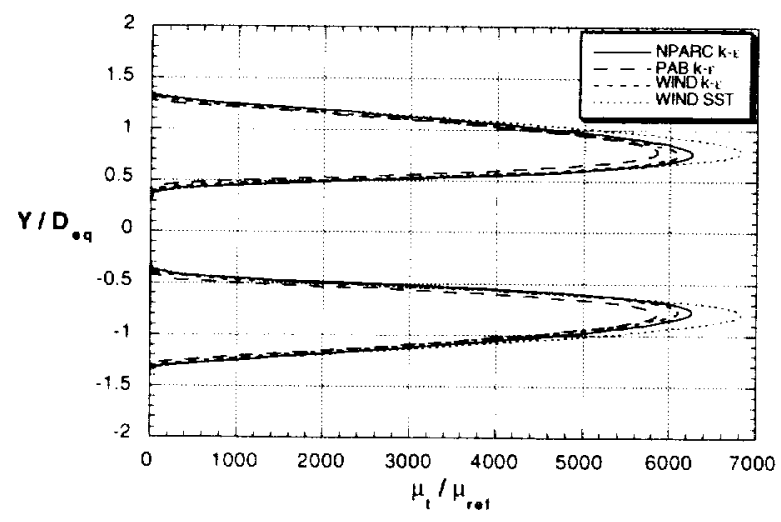

(a) $\mathrm{X} / \mathrm{D}_{\mathrm{eq}}=3.8$.

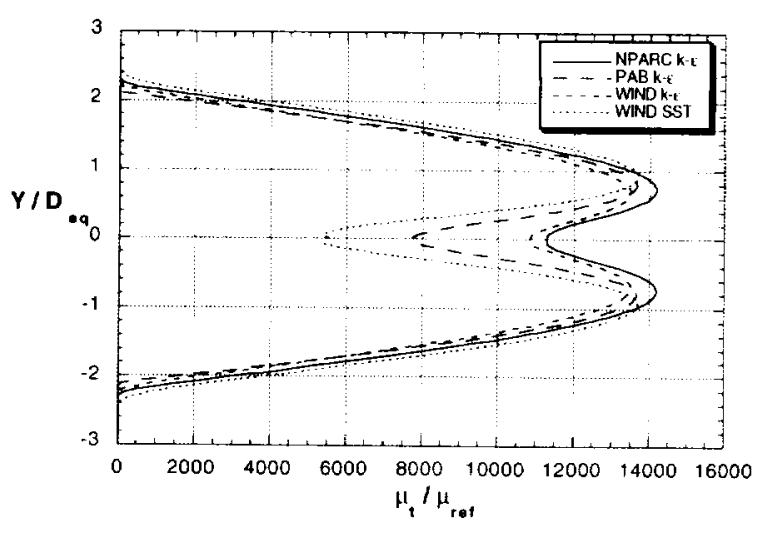

(b) $\mathrm{X} / \mathrm{D}_{\mathrm{eq}}=10.1$.

Fig. 7. Turbulent viscosity profiles along major axis for elliptic nozzle.

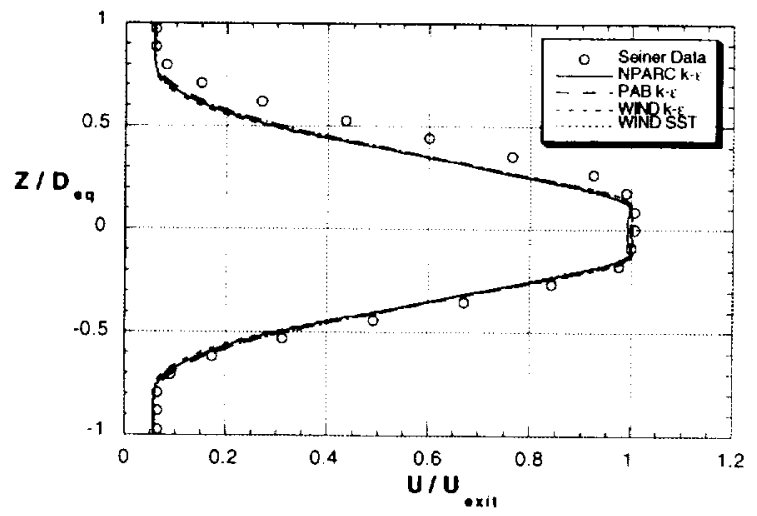

(a) $\mathrm{X} / \mathrm{D}_{\mathrm{cq}}=3.8$

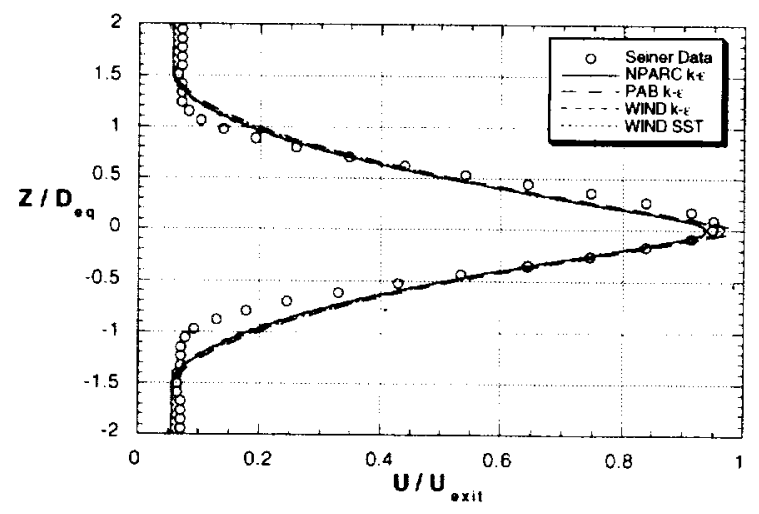

(c) $\mathrm{X} / \mathrm{D}_{\mathrm{c} 4}=7.6$.

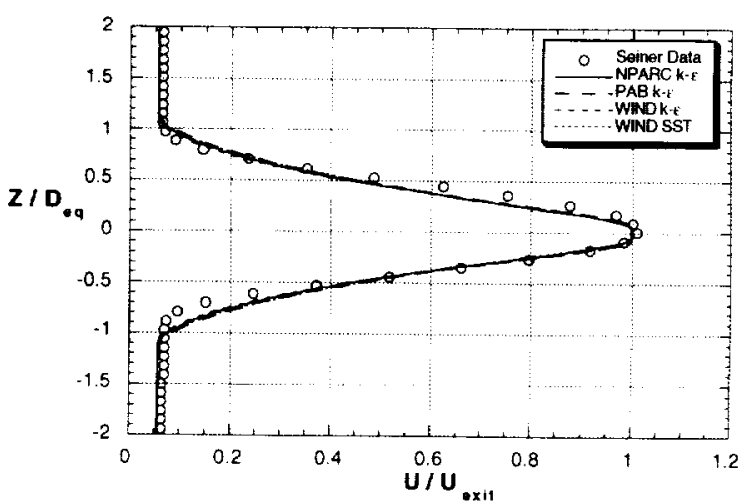

(b) $\mathrm{X} / \mathrm{D}_{\mathrm{cu}}=5.1$.

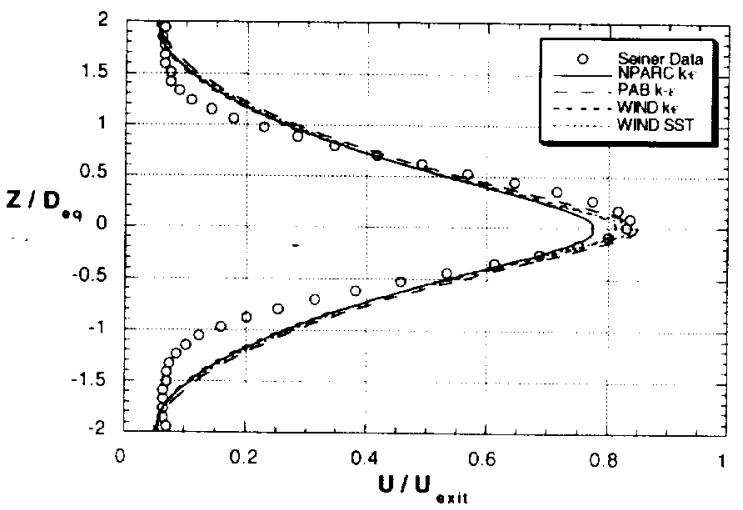

(d) $X / D_{c y}=10.1$.

Fig. 8. Velocity profiles along minor axis for elliptic nozzle. 


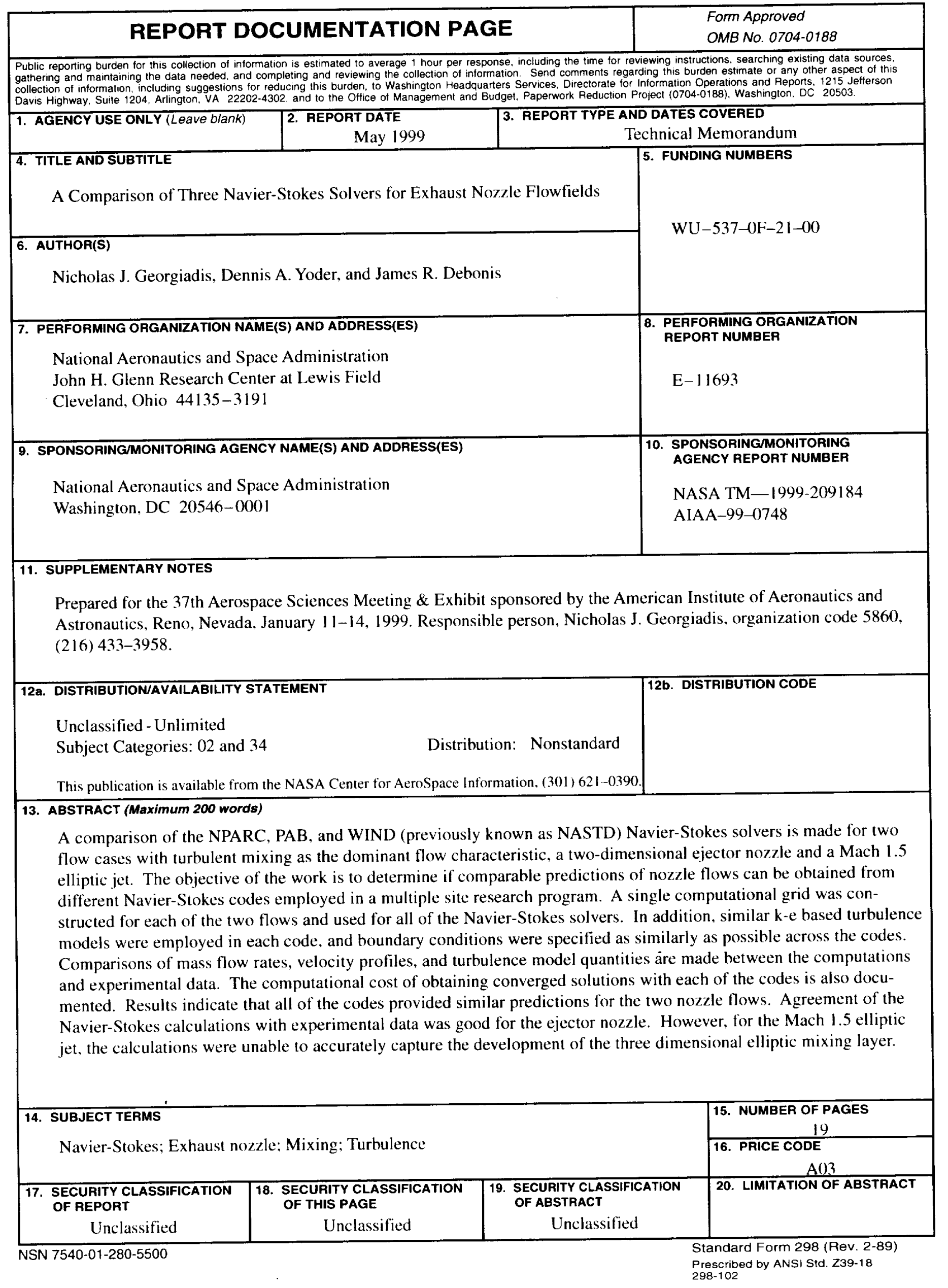




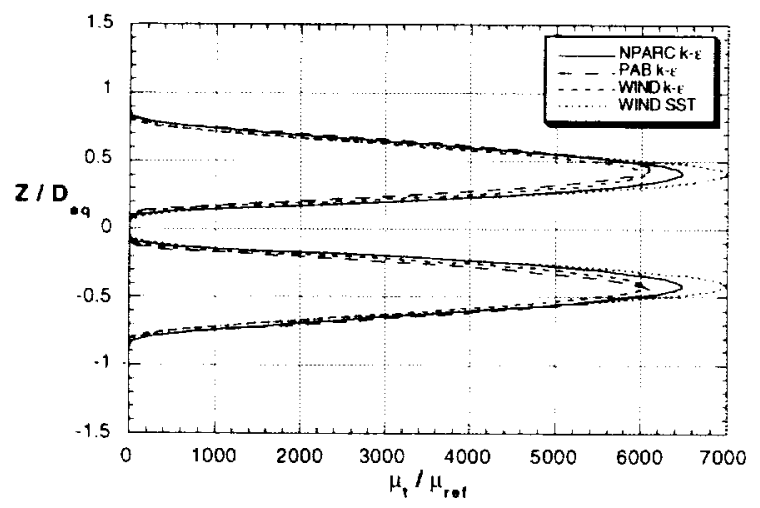

(a) $\mathrm{X} / \mathrm{D}_{\mathrm{ry}}=3.8$.

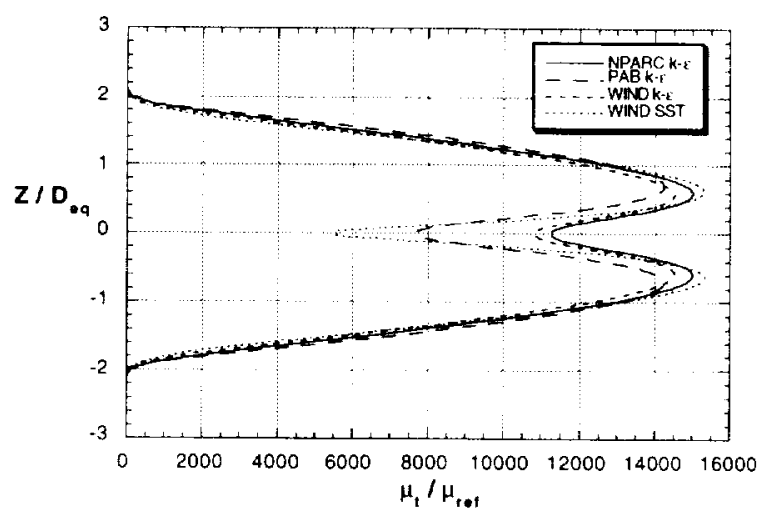

(b) $\mathrm{X} / \mathrm{D}_{\mathrm{e} 4}=10.1$.

Fig. 9. Turbulent viscosity profiles along minor axis for elliptic nozzle.

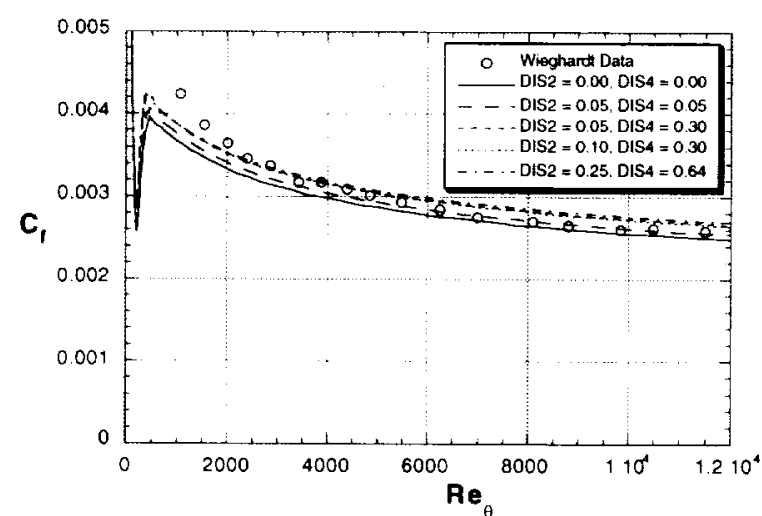

Fig. 10. Effect of NPARC artificial dissipation on houndary layer skin friction predictions.

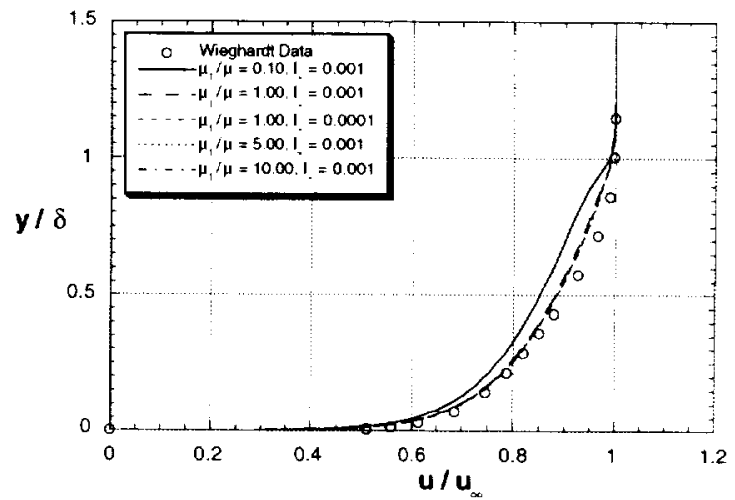

Fig. 11. Effect of PAB turbulence model limits on boundary layer velocity predictions.

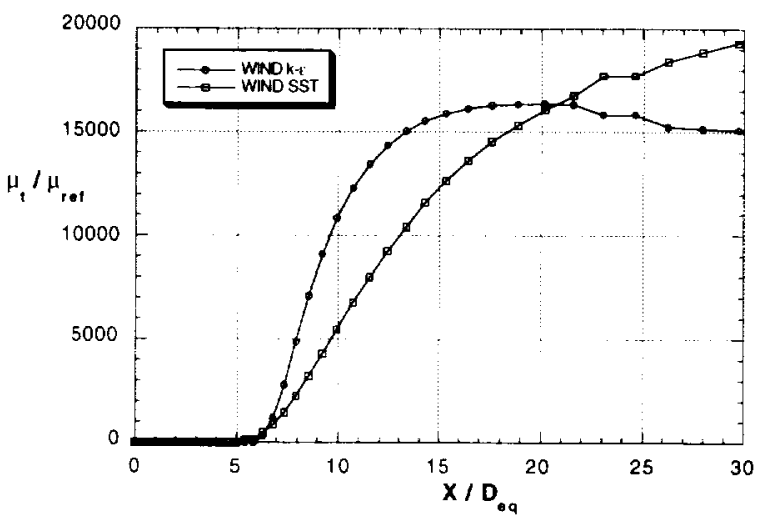

Fig. 12. Centerline turbulent viscosity for elliptic nozzle predictions with WIND. 